\title{
Chronic Chopart's joint dislocation and its treatment with the llizarov external fixator: a case report and literature review
}

\author{
Omer A.R. Barawi ${ }^{1}$, Soran S. Raoof ${ }^{2}$, Abd Ali Muhsin ${ }^{3}$ \\ ${ }^{1}$ College of medicine, University of Slemani, Kurdistan Regional, Iraq \\ ${ }^{2}$ Slemani Health Office, Iraq \\ ${ }^{3}$ College of medicine, University of Al-Nahrain, Baghdad, Iraq
}

\begin{abstract}
Background Chronic Chopart dislocation is one of the causes of acquired painful flat foot, which is treated by midtarsal arthrodesis causing limitation of movement and smaller-sized foot. Gradual reduction based on the principles of arthrodiastasis using the Ilizarov external fixator is used for treating chronic Chopart dislocation. The case and method Twenty-two yearold male presented with painful right flat foot fourteen months after a motor vehicle accident. Gradual reduction was used for the chronically dislocated Chopart's joint by arthrodiastasis using the Ilizarov external fixator. Result The follow-up result after four years is presented. The longitudinal arch of the foot recovered and the foot is painless with full range of movements; the size of the foot is preserved. Conclusion Treatment of chronic Chopart dislocation by arthrodiastasis using the Ilizarov external fixator is a preferred method of treatment as the size of the foot will be preserved, movement of the joint will not be restricted, the joint will be painless. There is no need for thromboprophylaxis, no chance of compartment syndrome and less operation time in comparison to arthrodesis.
\end{abstract}

Keywords: Chronic Chopart dislocation, Chopart's joint, Arthrodiastasis, Acquired flat foot

\section{INTRODUCTION}

The talonavicular and calcaneocubiod joints are commonly known as the mid-tarsal joint or Chopart's joint [1]. The Chopart's articular space was used by Francois Chopart, a French surgeon (1743-1795), as a practical space for amputation in cases of distal foot tumor [1-6]. The treatment priorities there should include addressing the injured soft tissue by immediate joint reduction or restoring bony alignment, avoiding the threat of compartment syndrome [1]. These fracture-dislocations are caused most commonly by motor vehicle accidents and fall from height and are frequent injuries in the polytrauma patients.

The most useful classification is that of Main and Jowett, which is based on the mechanism of injury $[1,7]$ :

- Medial stress injury

- Longitudinal stress injury

- Lateral stress injury

- Plantar stress injury

- Crush injuries

Failure of the talonavicular joint (TNJ) leads to flattening of the longitudinal arch, abduction of the forefoot and gradual valgus deformity of the subtalar joint and results in the painful flat foot [8]. The medial column of the foot consists of the talus, navicular bone, three cuneiform bones and the first, second and third metatarsal bones. The lateral column comprises the cuboid and the fourth and fifth metatarsal bones. The tarsal, navicular and cuboid bones are the cornerstones of the medial and lateral columns, respectively [9]. Recently, the use of distraction was described for treatment of ankle osteoarthritis. With this technique, the ankle is distracted using a small wire external fixator. Weight bearing is allowed. Due to a hinge or flexibility of the wires, intermittent fluid pressure is created within the joint; at the same time, the actual mechanical loading of the joint is avoided. Intermittent fluid pressure results in an increased proteoglycan synthesis in osteoarthritic joint. Intermittent fluid pressure has been associated with a decrease in the production of catabolic cytokines, interleukine-1 and tumor necrosis factor [10].

\section{CASE REPORT AND METHOD}

Twenty-two year old boy, $168 \mathrm{~cm}$ tall, his body weight of $70 \mathrm{~kg}$, presented to my clinic five years ago with complains of right foot pain, stiffness and flat foot for 14 months after motor vehicle accident. 


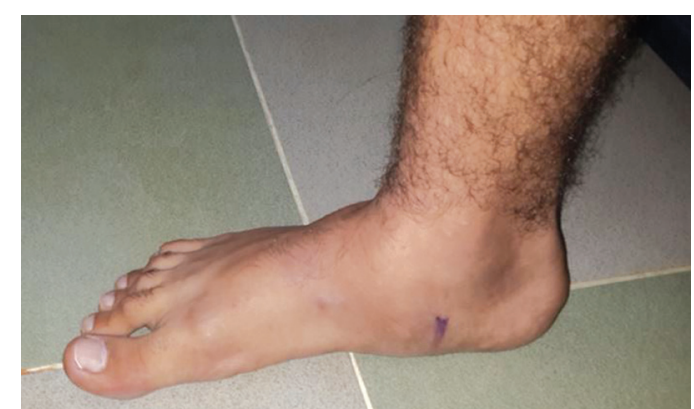

Fig. 1 Flattened longitudinal arch of the right foot (acquired flat foot)

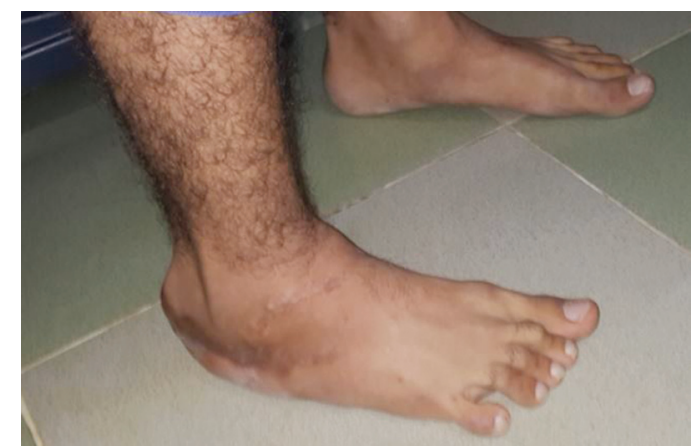

Fig. 2 Scars of previous trauma and operations

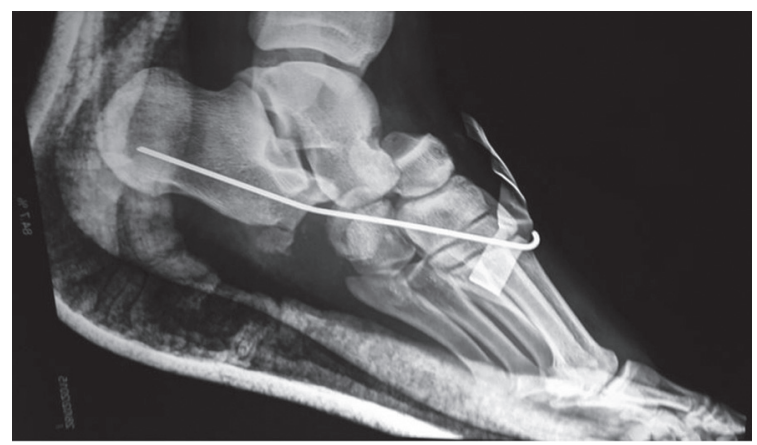

Fig. 3 Plain radiograph after an attempt of reduction, lateral view, reveals dislocated Chopart's joint

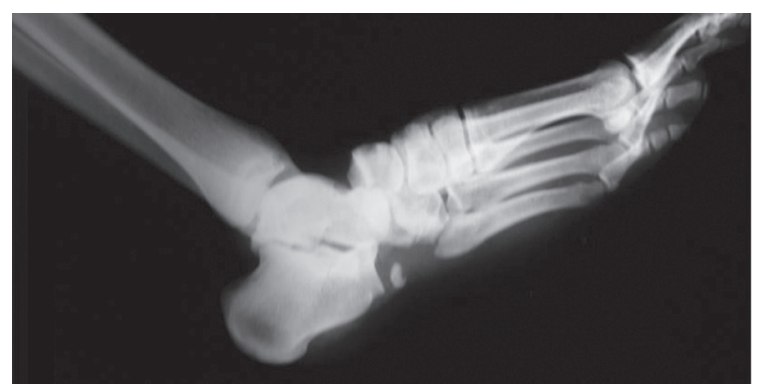

Fig. 4 At time of presentation, dislocated Chopart's joint

Procedure This novel technique can be performed under general or regional anesthesia, without open reduction, after the application of the Ilizarov frame which consists of a vertical full ring fixed to the forefoot neck of the metatarsals with two olive wires while a horizontally placed half-ring is fixed to the calcaneus with two olive wires. The two supports are connected with two rods. Daily distraction is $1 \mathrm{~mm}$, divided in four doses till dis-impaction of midtarsal joint is achieved. But the forefoot is translated dorsally, so the drooped olive wire is used to bring the navicular bone opposite to talus. The olive wire is held by two male supports, one of them is dorsally fixed to the ring while the second one is plantar, fixed to the ring (Fig. 5, 6).

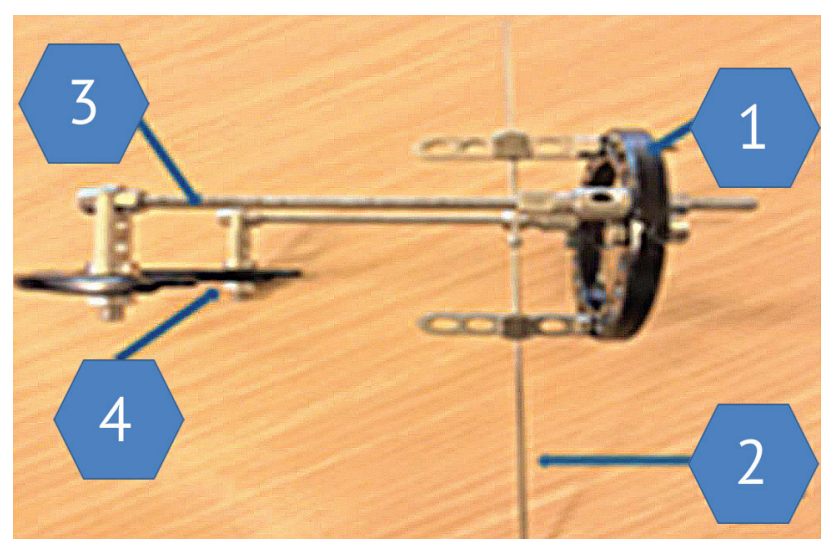

Fig. 5 The Ilizarov frame: 1 - Vertically located full ring to forefoot; 2 - Dropped wire to prevent upward translation of forefoot; 3 - Distracting rods; 4 - Horizontally located half ring for calcaneus

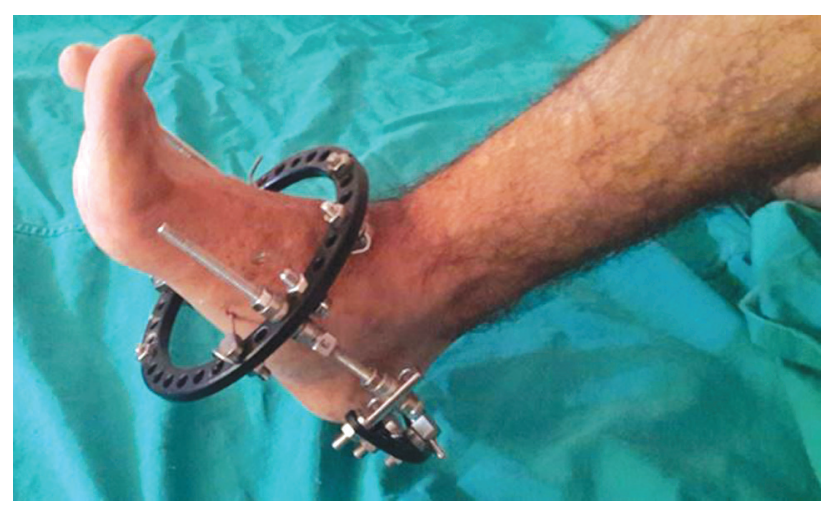

Fig. 6 Ilizarov external fixator is applied

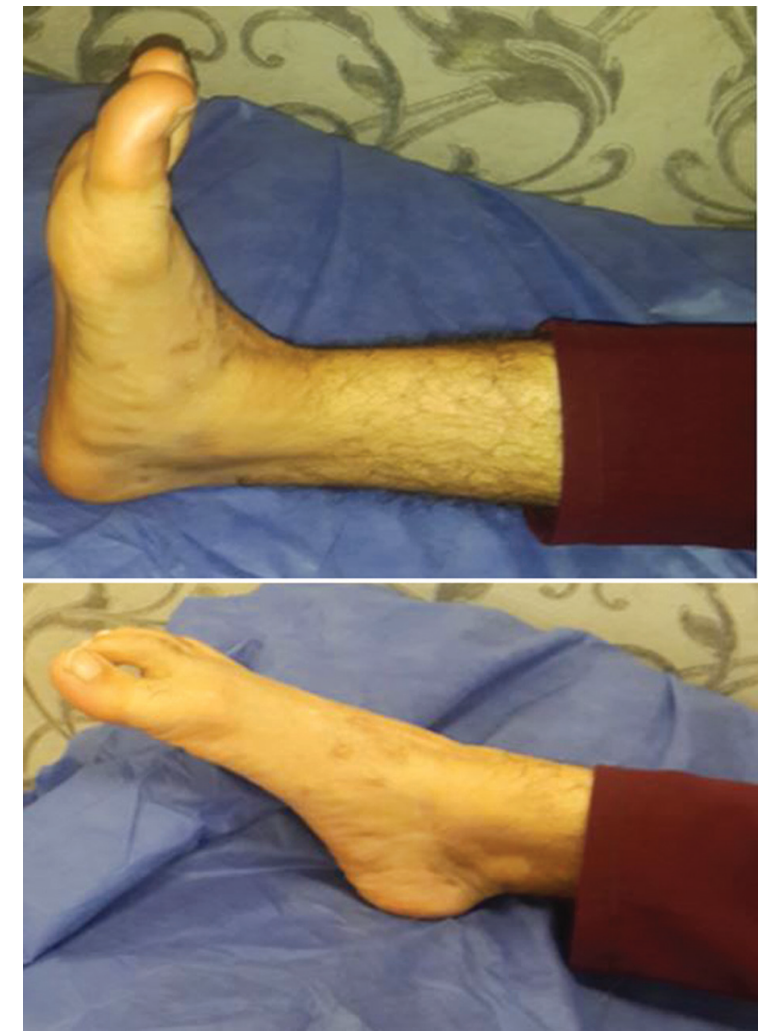

Fig. 7 After removal of the frame 


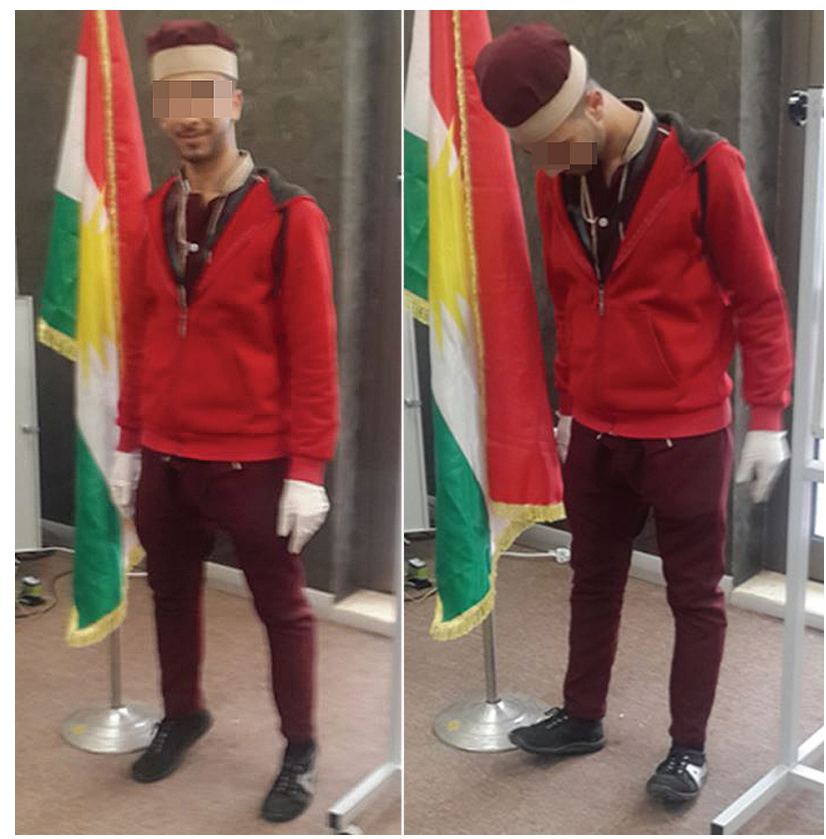

Fig. 8 One year after removal of the Ilizarov frame

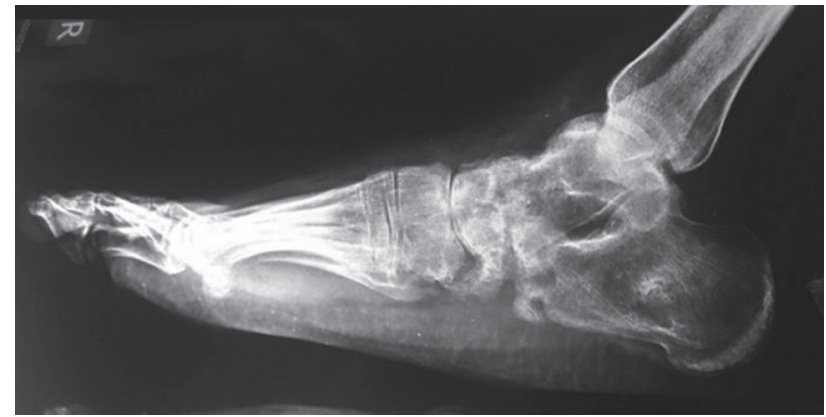

Fig. 9 Plain radiograph, lateral view, reveals well reduced Chopart's dislocation

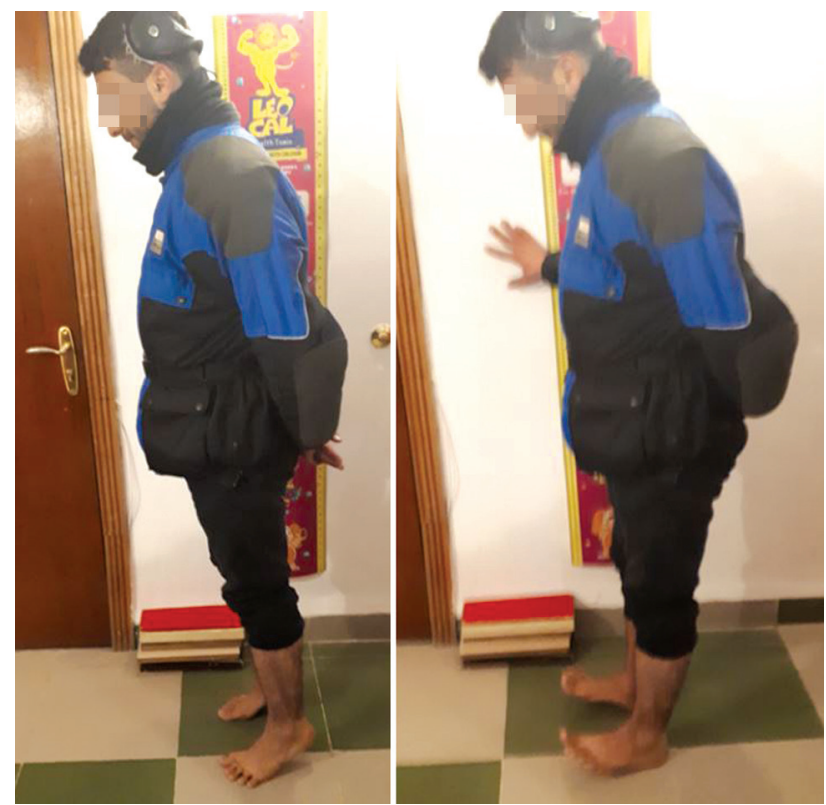

Fig. 10 Four years after removal of the Ilizarov frame; the patient is able to stand on tiptoes and heel

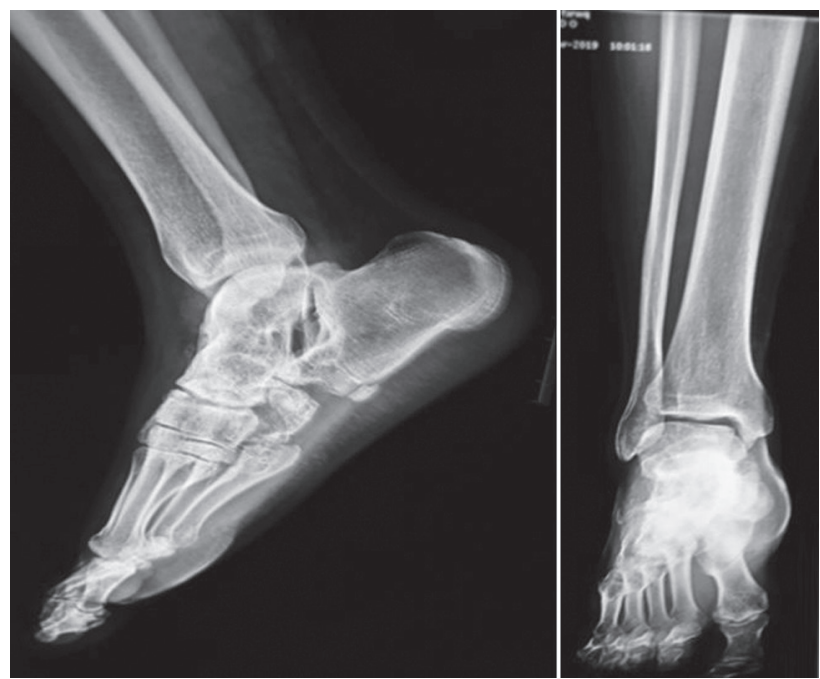

Fig. 11 Recent radiograph, 4-year follow-up, no evidence of disuse osteoporosis
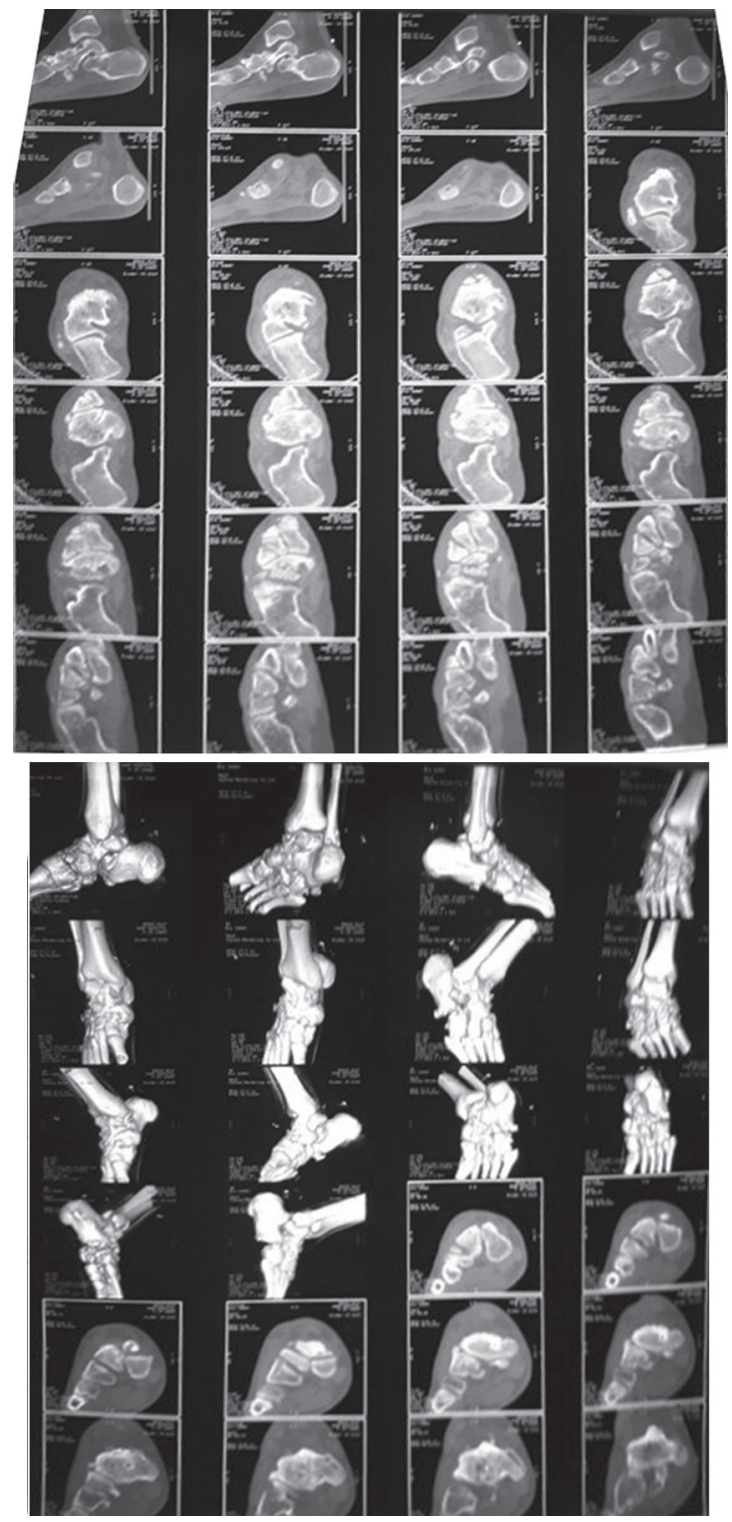

Fig. 12 Recent CT scan

\section{DISCUSSION}

After acute phase of the injury and insufficient articular reconstruction, salvage procedures include osteotomies and arthrodesis. Fusing the talonavicular joint involves locking the talo-calcaneo-navicular joint. 
A current accepted treatment for chronic dislocation of the Chopart's joint is arthrodesis, however it results in a significant loss of normal hindfoot motion $[1,3$, $6,8]$. We treated our case by gradual reduction of the dislocated Chopart's joint using the Ilizarov frame, based on the principle of joint distraction .The arterial anatomy is critical because the anterior tibial artery has an intermetatarsal branch, which has anastomosis with plantar circulation. Rupture of this anastomosis can cause major hemorrhage and compartment syndrome. The anastomosis may be damaged with repeated closed reduction maneuvers of strong force [11]. The merit of our method is gradual reduction by $1 \mathrm{~mm}$ daily in 4 divided doses for five days postoperatively done by the patient for 20 days.

We did not use thromboprophylaxis in our case as the leg and ankle joint are not included in the Ilizarov frame in contrast to some other methods [3].

The talo-navicular joints belong to the talocalcaneo-navicular joint (coxapedis), which is essential for pronation and supination of the whole foot [6]. In our case, we preserved these joints, so pronation and supination of the foot also remained preserved.
A new technique for treatment of fracturedislocation of the Chopart joint is using pre-tensioned hamstring autologous graft for reconstruction of the medial ligamentous complex (MLC) after fixation of all bony pathology. This joint-sparing method of reconstruction provides excellent clinical and functional outcome, surpassing the previously reported in the literature. Thromboprophylaxis should be performed in accordance with local guidelines [3]. The use of external fixator for ankle and foot injuries is less invasive, can prevent complications and more favorable than internal fixation which may cause excessive invasive second surgery, plate infection and system failure. Moreover, there are other advantages of using external fixator such as a decrease in the operative time and amount of soft-tissue dissection. The external fixation can provide neutralization and rigid stabilization, adjustable amounts of distraction, compression, rotation and translation as necessary during the treatment [12].

In our case, we used the American Orthopedic Foot and Ankle Society (AOFAS) hindfoot score for assessment, which scored a total of 100 points out of 100 .

\section{CONCLUSION}

Treatment of chronic Chopart's dislocation by joint distraction using the Ilizarov external fixator has the following advantages:

1. Mobility of the joint will be preserved as there is no joint fusion.

The authors do not declare any conflict of interest.

2. Size of the foot will be preserved as there is no resection of any articular surfaces.

3. No chance of compartment syndrome.

4. No need of thromboprophylaxis.

5. Shorter duration of operation in comparison to arthrodesis.

\section{REFERENCES}

1. Klaue K. Treatment of Chopart Fracture-Dislocations. Eur. J. Trauma Emerg. Surg., 2010, vol. 36, no. 3, pp. 191-195. DOI: 10.1007/s00068-010-1047-0.

2. Khan A.P., Raffic M. Chopart's Fracture-dislocation - rare Cases Management. Kerala Journal of Orthopedics, 2018, vol. 31, no. 1, pp. 31-34.

3. Kadakia A.R., Ho B.S., Molloy A. A New Technique of Treatment of Fracture-Subluxation of Chopart Joint. Techniques in Foot \& Ankle Surgery, 2013, vol. 12, no. 4, pp. 201-209. DOI: 10.1097/BTF.0b013e3182a001e0.

4. Van Dorp K.B., de Vries M.R., van der Elst M., Schepers T. Chopart joint injury: a study of outcome and morbidity. J. Foot Ankle Surg., 2010, vol.49, no. 6, pp. 541-545. DOI: $10.1053 /$ j.jfas.2010.08.005.

5. Rammelt S., Schepers T. Chopart Injuries: When to Fix and When to Fuse? Foot Ankle Clin., 2017, vol. 22, no. 1, pp. 163-180. DOI: 10.1016/j. fcl.2016.09.011.

6. Kale D.R., Khadabadi N.A., Putti B.B., Jatti R.S. One Month Old Neglected Lisfrancs Fracture Dislocation Treated with Wagner's External Fixator and Percutaneous Screw Fixation: A case report. J. Orthop. Case Rep., 2014, vol. 4, no. 2, pp. 42-46. DOI: 10.13107/jocr.2250-0685.166.

7. Blom A., Warwick D., Whitehouse M. Apley and Solomon's System of Orthopaedics and Trauma. Tenth Edition. CRC Press, 2017, pp.958-960.

8. Mansour A., Fallat L. Temporary bridge plating of the medial column in Chopart and Lisfranc injuries. The Foot and Ankle online Journal, 2017, vol. 10, no. 1, p. 5. doi:10.3827/faoj.2017.1001.0005.

9. Tse M. S.-H., Woo S.-B. Temporary Bridging of Severe Medial Column Injury of the Foot using Internal Fixation. Journal of Orthopaedics,Trauma and Rehabilitation, 2014, vol. 18, no. 2, pp. 120-123. DOI: 10.1016/j.jotr.2013.09.001.

10. Chiodo C.P., McGarvey W. Joint distraction for the treatment of ankle osteoarthritis. Foot Ankle Clin., 2004, vol. 9, no. 3, pp. 541-553. DOI: 10.1016/j.fcl.2004.05.007.

11. Richter M., Thermann H., Huefner T., Schmidt U., Goesling T., Krettek C. Chopart joint fracture-dislocation: initial open reduction provides better outcome than closed reduction. Foot Ankle Int., 2004, vol. 25, no. 5, pp. 340-348. DOI: 10.1177/107110070402500512.

12. Ramlee M.H., Derus AA. The use of external fixator for ankle and foot injuries management - a review on biomechanical perspective. Med. Dev. Diagn. Eng., 2016, vol. 1, no. 1, pp. 5-10. DOI: 10.15761/MDDE.1000102.

Received: 21.01.2020

\section{Information about the authors:}

1. Omer A.R. Barawi, Dr., Professor,

College of medicine, University of Slemani, Kurdistan Regional, Iraq,

Email: o_barawi@hotmail.com

2. Soran S. Raoof, Dr.,

Slemani Health Office, Iraq

3. Abd Ali Muhsin, Dr., Professor,

College of medicine, University of Al-Nahrain, Baghdad, Iraq 